\title{
The Biological Effectiveness and Medical Significance of Far Infrared Radiation (FIR)
}

\author{
Li-Chi Lin', Yi-Ying Lin ${ }^{2 *}$
}

${ }^{1}$ CEO, GHI FU Technology Co., Ltd., TAIWAN

${ }^{2}$ Founder, Green Luminant International Co., Ltd., TAIWAN

*Email for Correspondence: yiying0724@gmail.com

\begin{abstract}
The electromagnetic waves constitute different wavelengths of light from which Far infrared (FIR) is beneficial for living cells. Extensive studies and trials have been conducted over the last two decades in multidimensional biological domains to identify its unlimited health benefits. FIR radiations improve the microcirculation of the human body, stimulate cell growth, penetrate through skin tissues noninvasively, create intramolecular vibrations create an overall healthy metabolism, which ultimately affects overall improved cardiac and metabolic activity. This phenomenon is used to explore different pathological conditions to identify their significance in the medical field. In this review, we explored the biological effectiveness and the medical significance of Far infrared radiation (FIR) in murine melanoma Cell Growth, Lymphedema, airborne viruses, Cardiac diseases, Wound healing and burns, Autonomic Activities, Hemodialysis, Allergic Rhinitis, Aesthetic medicine, textiles, and other domains such as obesity and gut microbiota.
\end{abstract}

Keywords: Far infrared radiation (FIR), Infra-red, Electromagnetic waves, Biological effectiveness, Medical significance

\begin{tabular}{l} 
Manuscript Received: 14 April 2021 \\
\hline $\begin{array}{l}\text { This article is is licensed under a Creative Commons Attribution-NonCommercial 4.0 International License. } \\
\text { Attribution-NonCommercial (CC BY-NC) license lets others remix, tweak, and build upon work non-commercially, and although the new works must also } \\
\text { acknowledge and be non-commercial. }\end{array}$
\end{tabular}

\section{INTRODUCTION}

The potential of the energy greatly relies on the temperature to emit from a particular material. The sun is the most vital example of radiating radio-magnetic rays with an external temperature of $6,000^{\circ} \mathrm{C}$ and about $15,000,000^{\circ} \mathrm{C}$ at the inside. These rays, when entering the atmosphere of earth, lost their radio magnetic potential. In case, an infinitesimal amount of these potential rays can cause a nonreversible DNA damage to the human epithelial surface and even cancer. The received sunlight on earth composed of $6 \%$ ultraviolet rays, $52 \%$ visible rays, and $42 \%$ infrared rays. This sunlight has unlimited potential benefits to the living cells, if use in a right way (Yamashita, 2012).

The wavelength of infrared (IR) electromagnetic radiation is $780 \mathrm{~nm}$ and $1000 \mathrm{um}$. Based on the wavelengths, IR is categorized into three major bands: Near-Infrared, Mid-Infrared, and Far-Infrared (FIR). The wave length of 50.0$1000.0 \mu \mathrm{m}$ categorized as FIR (Tsai \& Hamblin, 2017; Vatansever \& Hamblin, 2012). An extensive literature based on the animal and human trials reported IR as a wound healing, preconditioning the skin, photo prevention, relief body tension/pain, exhaustion of rheumatoid arthritis, positive therapeutic effect on inflammatory diseases such as ankylosing spondylitis, cancer management by potentiating photodynamic therapy, and help to reduce optic, sensory, Autonomic activities, and psychological complaints. FIR is also beneficial to vitalize heart and mesenchymal stem cell functions and as a supportive management approach in aesthetic medicine (Yamashita, 2012; Tsai \& Hamblin, 2017; Vatansever \& Hamblin, 2012; Barolet et al., 2016; Cristiano, 2019; Yang et al., 2017). FIR Reflecting Textiles and 
clothing are other promising approaches to improve the functional abilities of the human body (Basuk, 2018). Recent studies reported additional emerging benefits of FIR, the deployment of FIR costumes in sports for better performance outcomes, and the inactivity of airborne microbial DNA (Bontemps et al., 2021; Li et al., 2020). Anticipation of Coronary heart disease also possible by the responsiveness of endothelial cells using FIR (Tsai et al., 2020).

\section{Statement of the Problem}

To resolve the FIR-related ambiguities and their health and medical effects, the tissue culture FIR incubator and animal breeding structure was designed. The carbon dioxide incubator certainly the perfect controlled environment example of FIR having $37 \pm 0.5^{\circ} \mathrm{C}$ temperature (Yamashita, 2012). The human body is largely composed of water molecules. The main concern related to FIR medical application is its interaction with water molecules. The important considerable features are ionic water interaction, dipole movement, and dielectric features. Another consideration of every living cell is its electromagnetic field. The cell proteins and charged particles are vital to cell components to balance the total cell activity. These charged particles also dependent on water molecules to attribute their dielectric behavior, which eventually responsible for the biological activity of every living cell. To conclude this, water is the most important content between FIR and living cell interaction. There are many peaks of large molecules in the FIR spectrum. There are six vibrating modes of $\mathrm{CH}_{2}$ compounds or organic compounds, i.e symmetric, antisymmetric stretching, scissoring, rocking, wagging, and twisting (Vatansever \& Hamblin, 2012).

Recent scientific experiments and literature find out that the biological activity of FIR is not dependent on cell thermal activities. However, no formal and genuine method yet defined to measure its non-thermal cellular and biological activities which identify the limitations and restrictions of the biological and medical effectiveness of FIR. However, animal trials are ongoing to identify the FIR biological index (FBI) and beneficial irradiation time by using the mitochondrial function of epithelial cells. No conclusive evidence yet identified (Hsu et al., 2019).

Table 1: Over view of the selected studies

\begin{tabular}{|l|l|l|l|}
\hline S. no & Author(s) & Year & Applications \\
\hline 1 & Leung et al. & 2012 & Murine Melanoma Cell Growth \\
\hline 2 & Li et al. & 2018 & Lymphedema \\
\hline 3 & Li et al. & 2020 & Instability of airborne viruses \\
\hline 4 & Beever & 2009 & Cardiovascular risk factors \\
\hline 5 & Tsai et al. & 2020 & Coronary artery disease \\
\hline 6 & Lin et al. & 2013 & Arteriovenous Fistula \\
\hline 7 & Lin et al. & 2015 & Wound Healing \\
\hline 8 & Simmons et al. & 2018 & Burn wound \\
\hline 9 & Yang et al. & 2017 & Autonomic Activities \\
\hline 10 & Su et al. & 2009 & Hemodialysis \\
\hline 11 & Hu et al. & 2007 & Allergic Rhinitis \\
\hline 12 & Chu et al. & 2015 & Allergic Rhinitis \\
\hline 13 & Suwandee et al. & 2014 & Cosmetics \\
\hline 14 & Cristiano & 2019 & Aesthetic medicine \\
\hline 15 & Basuk & 2018 & Textiles \\
\hline 16 & Bontemps et al. & 2021 & Sports garments \\
\hline
\end{tabular}

In this comprehensive review we will summarize the key findings of the scientific literature, specifically published in the last decade to explore and implement the new FIR medical applications and their biological effectiveness. An extensive literature search was conducted to find out the development of FIR in biology field and the significance of its medical significance. Table 1 presented the overview of selected studies.

\section{EMERGING FIR MEDICAL APPLICATIONS AND ITS BIOLOGICAL EFFECTIVENESS}

\section{Effect of FIR in Chronic Diseases}

Lymphedema is a life-threatening pathology characterized by the accumulation of body fluid, particularly in the legs. FIR radiation thermotherapy (FIRT helps tissue fibrosis in Lymphedema patients. A study was conducted in 2015 \& 2016 participated by stage II-III lymphedema patients for 20 sessions of FIRT therapy. The study reported a quite satisfactory outcome in improving tissue fibrosis and epithelial elasticity, affecting the overall quality of life (Li et al., 2018). An evaluation was done by using skin elasticity, which improved by $p<0.05$, ultrasound testing for fibrosis status, and laboratory testing for fluid concentration. The improvement was seen in reducing tension, heftiness, pain, numbness, firmness, and discomfort ( $\mathrm{Li}$ et al., 2018). Another study reported promising outcomes in Murine 
melanoma cells, particularly B16- F10 by using somatothermal cFIR. The FIR application reduce the melanoma cells proliferation, intracellular nitric oxide, and heat shock protein by $11.8 \%, 15.7 \%$, and $56.9 \%$ after $48 \mathrm{hrs}$ of exposure (Leung et al., 2012). Somatothermal cFIR strategy represses the B16-F10 proliferation and shuts down the production of nitric oxide and HSP70. Intracellular Reactive oxygen species also produced due to cFIR cell exposure, which leads to cell cycle disturbance and ultimately growth arrest (Li et al., 2018). However, this research domain required further large scale research to find out the standard cFIR strategy for management of chronic diseases.

\section{Instability of airborne viruses}

Airborne microorganisms are great threat to human beings and the recent pandemic of SARS-CoV-2 is its classical example. Studies conducted to explore the effect of FIR to control these airborne micro-organisms which are around everywhere to cause life-threatening respiratory infections. Far infrared (FIR) heating fans are the accessible product to warm the closed environments. These FIR heating fans emit electromagnetic radiation between 5.6-1000 $\mu \mathrm{m}$ ( $\mathrm{Li}$ et al., 2020; Shui et al., 2015). These radiations disturbed the nucleic acids of DNA and RNA viruses (Li et al., 2020). DNA viruses won't significantly damage by FIR radiations. However, significant degradation of RNA viruses was reported as a result of FIR heating fan exposure. Four-hour duration of 400 wattages of FIR heating fans in a $51 \mathrm{~m}^{3}$ room area was recommended. Using these Far infrared (FIR) heating fans is a reliable and cheap method to reduce the flow of infectious RNA airborne viruses ( $\mathrm{Li}$ et al., 2020). Most life-threatening air-borne viruses are RNA viruses including influenza, Severe acute respiratory syndrome (SARS), and SARS, CoV-2, which makes this strategy the most appropriate to adapt (Li et al., 2020; Shui et al., 2015).

\section{Reducing Cardiovascular risk factor}

An extensive review was conducted in the last decade to analyze the effect of FIR ranging from $0.75-1000 \mu \mathrm{m}$ to improve cardiac health. Congestive heart failure (CHF), coronary risk factors, chronic pain, chronic fatigue syndrome were the analyzed pathological conditions. The most adapted method was an infrared sauna that reportedly improves ventricular arrhythmias and endothelial function to reduce the risk of congestive heart failure and improve overall cardiac health (Beever, 2009). The overall response of FIR saunas was very effective for cardiac health based on different randomized control trials. However, these studies did not conclude the recommended exposure time, measurement of basic parameters before and after exposure such as blood pressure, heart rate, etc. Some studies rehydrated the participants before a day of collecting post-treatment parameters, such as hematocrit, glucose, cholesterol, catecholamine, and triglyceride values (Beever, 2009). A recent study further explores this benefit and identify that miR-548aq-3p is the distinctive target for the prediction of heart disease by using Far infrared radiation (Tsai et al., 2020). miR-548aq-3p target not yet identified as any biological role, it was explored that this novel target may enhance the functionality of Endothelial colony-forming cells after FIR radiation. These Endothelial colonyforming cells responsible for the vascular restoration and coronary artery disease (Tsai et al., 2020).

\section{Arteriovenous Fistula and FIR therapy}

Arteriovenous Fistula malfunctioning occurs because of multiple genetic, pathological, and mechanical reasons. The development of Arteriovenous Fistula is a major cause of patients with chronic renal disease. A study was conducted by using FIR WS TY101N technology to evaluate the FIR efficiency in chronic renal disease - Arteriovenous Fistula patients. The promising results were reported, and FIR greatly improves the Arteriovenous Fistula in stage IV and V renal disease patients (Lin et al., 2013). Vasodilatation was created due to the thermal reaction of FIR radiation. The FIR radiation was provided up to $2-3 \mathrm{~cm}$ deep of the skin surface by increasing the temperature to $38^{\circ} \mathrm{C}-39^{\circ} \mathrm{C}$. The exposure time of $30-$ 60minutes was recommended to conduct this process, 3 times a week up to 1 year (Lin et al., 2013). The initial therapeutic benefits were on the severity of disorder and rate of embolism and presence of clots (Lin et al., 2013).

\section{Wound Healing and Burns}

Wound healing by FIR application is one of the crucial domains of FIR applications. Initially, there was an ambiguity that FIR did not participate in wound healing. Later on, many trials were conducted to find out that after exposure to FIR radiations the blood flow of the exposed wound area was increased. Many scientific researches were conducted on animal models initially to affirm the research concept and identified the gene expression of TGF-b1 at the wound site for healing. These studies find out that FIR radiation promotes the biological activities of the wound area including blood circulation, proliferation of fibroblast, collagen formation, and TGF-b1production (Lin \& Li, 2017; Toyokawa et a., 2003; Yu et al., 2006).

The phenomenon further explored for burn wounds, and studies conducted on extreme superficial burns to identify the decision of skin graft. Skin grafting is the most suggested management in most extreme burn wounds. However, the clinical examination is deficient to conclude the management decision. The FIR radiations helped in burn wounds to identify the viability of the burn site, which is quite difficult by physical examination. FIR technique is simple to explore the true diagnosis in burn wound and decision of grafting (Simmons et al., 2018). FIR camera - Therm-App ${ }^{\circledR}$ 
camera use to execute this phenomenon by taking images of burn site up to 2 minutes in every 5 seconds. Total of 24 images collected in 2 minutes. After grafting, the same process applied to identify the progress of burn wounds in deep cells. This FIR technique reportedly the most precise technique to identify the true status of burn wounds, its grafting decision, and progress after grafting (Simmons et al., 2018).

\section{Far-Infrared and Acupuncture therapy to improve Autonomic Activities and Stroke}

Having multiple benefits of FIR in wound healing, and improvement in cardiac health by engaging fibroblast and increase blood circulation, its role in autonomic activities were explored in combination with acupuncture, which is the traditional management approach for many disorders. The combined management strategy of using acupuncture and FIR radiation was reportedly highly beneficial in cardiac disorders, metabolism, hypertension, and stroke (Yang et al., 2017; Hu \& Li, 2007).

Animals and human trials have been conducted to find out the FIR application in brain injury and stroke patients. However, these studies were inconsistent in the last decade but yet have a promising future. Transcranial near-infrared light therapy (NILT) was the adapted application to cure brain injury in stroke patients. NILT creates neuro regenerative modification by enhancing adenosine triphosphate (ATP) release, DNA replication, gene response, promote expression of growth factors, cell proliferation, and synaptogenesis (Hu \& Li, 2007; Henderson, 2016).

\section{Allergic Rhinitis}

Allergic rhinitis (AR) is a heterogeneous chronic respiratory disorder; ranked sixth among the most reported chronic complaints globally and greatly affects patient's wellbeing and quality of life. The management of AR is quite complex which greatly affects patient's daily life due to additional precautions and medications. The identification of allergen, allergen prevention, pharmacological medicines, and immunotherapeutic are the routine management requirements of AR. FIR radiations by using WS TY101FIRemitter to the nasal area of the affected patient were significantly helpful. The outcome depends upon the illness severity by improving irritation of eyes and nose, sneezing, and running nose (Su et al., 2009; Cho et al., 2015). This treatment is significantly helpful to a large population group, especially in seasonal allergens (Su et al., 2009; Cho et al., 2015).

\section{Cosmetics and Aesthetic medicine and FIR application}

Having unlimited health benefits of FIR on human cells, cosmetics, and aesthetic medicine also took advantage of these radiations to provide a healthy, reliable, and affordable beauty solution. Sauna blankets one of the most productive aesthetic approaches of FIR. FIR Sauna therapy is quiet promising for chronic pain relief, chronic fatigue syndrome, and weight loss due to neurohormonal cell response (Biro et al., 2003; Masuda et al., 2005a; Masuda et al., 2005b). There are unlimited FIR cosmetic products are available; such as FIR blankets, soft lasers, FIR lamps, FIR caps, and FIR helmets (Cristiano, 2019; Suwandee \& Yupapin, 2014). All these tools had medicinal benefits on human cells, both localized and systemic depending upon the tool used. Removal of fat promotes hair growth and treating alopecia are some of its aesthetic benefits (Cristiano, 2019). The aesthetic FIR devices are also available in markets for home use such as FIR hair brushes to prevent hair loss and hair shining. FIR lamps and FIR pens are also easily available. FIR pens used for pain relief on certain points such as the neck or back. There are unlimited beauty products are available which used FIR radiations, to make their product more beneficial for humans (Cristiano, 2019; Suwandee \& Yupapin, 2014).

\section{Effect of Infrared-emitting textiles and potential of FIR garments in sports}

FIR domain is greatly explored by technology advancement. The technology of nanoparticles used to impregnate textile fibers with FIR radiations. These garments used to obtain numerous health advantages and well-being of humans (Basuk, 2018). This textile development use radiations of $700 \mathrm{~nm}$ to $1 \mathrm{~mm}$. The fabrics designed by using FIR emitting potential, designed by adding IR potent trace elements, metals, and ceramics. The main characteristics to choose a suitable component for FIR fiber designing are high heat retention and the ability to absorb and reserve heat. The reverse mechanism applied in a cold environment, the heat generated by human cells preserve in these fiber components to prevent heat loss and make you feel warm (Basuk, 2018). This heat shifting is the basic mechanism of FIR textiles. Based on this, FIR textiles designate into different groups such as:

- Passive textiles to regulate the temperature of the human body, either way, warm or cold,

- Active warming/cooling textiles to add additional warm or cool input.

Responsive textiles for personal management of thermal indicators such as temperature, moisture, and $\mathrm{pH}$ of the human body (Peng \& Cui, 2020; Farooq \& Peng, 2021). Almost all clothing forms of FIR radiating fibers are available, with the specification of clothing insulation measure. This clothing insulation information based on the heat retention capacity and controls the thermal balance of the human body (Basuk, 2018). These FIR garments demand by elderly people and urban residents (Basuk, 2018). 
Manufacturing of sports, garments is an additional domain in sports textiles for performance optimization and better outcome of the sports person. The athletes and gymnasts, because of countless related benefits, had widely accepted compression garments but is it a reality or fad, is still an exploring doubt (Beever, 2009). An extensive review recently published by Bontemps et al. (2021) to clarify the related uncertainty (Beever, 2009). The study concludes that these sports garments generate a better productivity outcome by controlling the human body's hemodynamic and thermoregulation activities (Beever, 2009). These sports garments, depending upon the fiber nature, control blood flow, body temperature, oxygen intake, Cardiac activity, serum lactate, Increase Creatinine kinase, body posture, sleep activity, etc. (Beever, 2009). These garments have an immiscible category in health improvement; still, there is a lot more to explore. Physiotherapy is a medical subcategory responsible for the mobility of human muscles and patients' better well-being (Putowski et al., 2016). There are many FIR apparatus for better patient outcome, used in physiotherapy such as lamps, Electrotherapy, Magnetic therapy, Phototherapy, etc. FIR garments are yet to explore in Physiotherapy (Beever, 2009).

\section{Other Biological Benefits}

FIR radiations have multi-dimensional potential in health care and medical. Animal trials have been conducted to explore its benefits in cell metabolism, obesity, and weight loss. These animal trials were quite promising to develop a management strategy of weight loss by using FIR radiations. FIR radiations reduce appetitive by modifying gene expression of TRPV3-POMC (Hu et al., 2011). Another animal study finds out the modification of GLUT-4 gene expression in response to FIR radiations to control obesity (Kokura et al., 2007). These gene modifications were happened due to the hypothermia caused by FIR radiations (Hu et al., 2011; Kokura et al., 2007). Another recent study on the animal models investigated the phenomenon of FIR impact on gut microbes. The findings suggested the gastrointestinal modulation by activating the Host's G-protein-coupled receptors (GPCR) (Khan et al., 2020).

\section{CONCLUSION}

FIR has unlimited health benefits and impact on the human body among all other electromagnetic waves, which are yet to explore. In this review, we explored the most recent scientific literature reported the significant medical applications of FIR radiations and their promising outcome. However, there is a great need to investigate its benefits in managing chronic diseases, and life-threatening medical threats like SARS-CoV-2.

\section{REFERENCES}

Barolet, D., Christiaens, F., Hamblin, M. R. (2016). Infrared and skin: Friend or foe. Journal of Photochemistry and Photobiology B: Biology, 155, 78-85, https://doi.org/10.1016/j.jphotobiol.2015.12.014

Basuk, M. (2018). Improving the Performance of Human Body with Far Infra-Red Rays Reflecting Textiles. Current Trends in Fashion Technology \& Textile Engineering, 4(3), 51-59.

Beever, R. (2009). Far-infrared saunas for treatment of cardiovascular risk factors: summary of published evidence. Can Fam Physician, 55(7), 691-696. https:// pubmed.ncbi.nlm.nih.gov/19602651/

Biro, S., Masuda, A., Kihara, T., \& Tei, C. (2003). Clinical Implications of Thermal Therapy in Lifestyle-Related Diseases. Experimental Biology and Medicine, 228(10), 1245-1249. https://doi.org/10.1177/153537020322801023

Bontemps, B., Gruet, M., Vercruyssen, F., Louis, J. (2021). Utilisation of far infrared-emitting garments for optimising performance and recovery in sport: Real potential or new fad? A systematic review. PLOS ONE 16(5), 1-15, https://doi.org/10.1371/journal.pone.0251282

Bontemps, B., Gruet, M., Vercruyssen, F., Louis, J. (2021). Utilisation of far infrared-emitting garments for optimising performance and recovery in sport: Real potential or new fad? A systematic review. PLOS ONE, 16(5), e0251282. https://doi.org/10.1371/journal.pone.0251282

Cho, H. K., Jeong, Y. M., Lee, H. S., Lee, Y. J., \& Hwang, S. H. (2015). Efficacy of Endonasal Phototherapy for Relieving the Symptoms of Allergic Rhinitis: Meta-Analysis. American Journal of Rhinology $\mathcal{E}$ Allergy, 29(4), 283291. https://doi.org/10.2500/ajra.2015.29.4190

Cristiano, L. (2019). Use of infrared as a complementary treatment approach in medicine and aesthetic medicine. Asp Biomed Clin Case Rep. 2(2), 77-81. https://doi.org/10.36502/2019/ASJBCCR.6164

Farooq, A. S., Peng, Z. (2021). Fundamentals, materials and strategies for personal thermal management by next-generation textiles. Composites Part A: Applied Science and Manufacturing, 142, 106249. https://doi.org/10.1016/i.compositesa.2020.106249

Henderson, T. A. (2016). Multi-watt near-infrared light therapy as a neuroregenerative treatment for traumatic brain injury. Neural Regen Res, 11(4), 563-565. https://doi.org/10.4103/1673-5374.180737

Hsu, YH., Chen, YW., Cheng, CY., Lee, SL., Chiu, TH., \& Chen, CH. (2019). Detecting the limits of the biological effects of far-infrared radiation on epithelial cells. Scientific Reports, 9, 1-9, https://doi.org/10.1038/s41598-019-48187-0

Hu, J., Choo, H. J., \& Ma, S. (2011). Infrared heat treatment reduces food intake and modifies expressions of TRPV3-POMC in the dorsal medulla of obesity prone rats. International Journal of Hyperthermia, 27(7), 708716, https://doi.org/10.3109/02656736.2011.601283 
Hu, KH., \& Li, WT. (2007). Clinical effects of far-infrared therapy in patients with allergic rhinitis. Annu Int Conf IEEE Eng Med Biol Soc., 1479-1482. https://doi.org/10.1109/iembs.2007.4352580

Khan, I., Pathan, S., Li, X. A., Leong, W. K., Liao, W. L., Wong, V., Hsiao, W. L. W. (2020). Far infrared radiation induces changes in gut microbiota and activates GPCRs in mice. Journal of Advanced Research, 22, 145-152, https://doi.org/10.1016/j.jare.2019.12.003

Kokura, S., Adachi, S., Manabe, E., Mizushima, K., Hattori, T., Okuda, T., Nakabe, N., Handa, O., Takagi, T., Naito, Y., Yoshida, N., \& Yoshikawa, T. (2007). Whole body hyperthermia improves obesity-induced insulin resistance in diabetic mice. International Journal of Hyperthermia, 23(3), 259-265, https:/ / doi.org/10.1080/02656730601176824

Leung, TK., Chan, CF., Lai, PS., Yang, CH., Hsu, CY., Lin, YS. (2012). Inhibitory Effects of Far-Infrared Irradiation Generated by Ceramic Material on Murine Melanoma Cell Growth. International Journal of Photoenergy, Volume 2012, Article ID 646845. https://doi.org/10.1155/2012/646845

Li, E., Lu, J., Dong, S., Zhang, M., Cen, S., Li, L. and Huang, W. (2020). Instability of Nucleic Acids in Airborne Microorganisms under Far Infrared Radiation. Health, 12, 998-1007. https://doi.org/10.4236/health.2020.128074

Li, K., Zhang, Z., Liu, N. F., Sadigh, P., Evans, V. J., Zhou, H., Gao, W., and Zhang, Y. X. (2018). Far-Infrared Radiation Thermotherapy Improves Tissue Fibrosis in Chronic Extremity Lymphedema. Lymphatic Research and Biology, 16(3), 248-257. https://doi.org/10.1089/lrb.2016.0057

Lin, CC., Yang, WC., Chen, MC., Liu, WS., Yang, CY., Lee, PC. (2013). Effect of far infrared therapy on arteriovenous fistula maturation: an open-label randomized controlled trial. Am J Kidney Dis., 62(2), 304-311. https://doi.org/10.1053/j.ajkd.2013.01.015

Lin, Y.-H., \& Li, T.-S. (2017). The Application of Far-Infrared in the Treatment of Wound Healing: A Short Evidence-Based Analysis. Journal of Evidence-Based Complementary E Alternative Medicine,22(1), 188. https://doi.org/10.1177/2156587215623436

Masuda, A., Kihara, T., Fukudome, T., Shinsato, T., Minagoe, S., Tei, C. (2005). The effects of repeated thermal therapy for two patients with chronic fatigue syndrome. J Psychosom Res, 58(4), 383-387.

Masuda, A., Koga, Y., Hattanmura, M., Minagoe, S., Tei, C. (2005). The effects of repeated thermal therapy for patients with chronic pain. Psychother Psychosom, 74(5), 288-294.

Peng, Y., \& Cui, Y. (2020). Advanced textiles for personal thermal management and energy, Joule, 4(4), 724-742. https://doi.org/10.1016/j.joule.2020.02.011

Putowski, M., Piróg, M., Podgórniak, M., Padała, O., Sadowska, M., Bazylevycz, A., \& Wdowiak, A. (2016). The use of electromagnetic radiation in the physiotherapy. Eur J Med Technol, 2(11), 53-58.

Shui, S., Wang, X., Chiang, J. Y., \& Zheng, L. (2015). RETRACTED: Far-infrared therapy for cardiovascular, autoimmune, and other chronic health problems: A systematic review. Experimental Biology and Medicine, 240(10), 12571265. https://doi.org/10.1177/1535370215573391

Simmons, J. D., Kahn, S. A., Vickers, A. L., Crockett, E. S., Whitehead, J. D., Krecker, A. K., Lee, YL., Miller, A. N., Patterson, S. B., Richards, W. O., Wagner, W. W. Jr. (2018). Early Assessment of Burn Depth with Far Infrared Time-Lapse Thermography. J Am Coll Surg., 226(4), 687-693. https://doi.org/10.1016/j.jamcollsurg.2017.12.051

$\mathrm{Su}, \mathrm{LH} ., \mathrm{Wu}, \mathrm{KD}$., Lee, LS., Wang, H, Liu, CF. (2009). Effects of far infrared acupoint stimulation on autonomic activity and quality of life in hemodialysis patients. The American Journal of Chinese Medicine, 37(2), 215-226. https://doi.org/10.1142/S0192415X09006783

Suwandee, S. \& Yupapin, P. P. (2014). Gemstone Property Studies for Minerals Based Cosmetics and Beauty Applications, Life Science Journal, 11(11), 871-873, https://doi.org/10.1016/j.joule.2020.02.011

Toyokawa, H., Matsui, Y., Uhara, J., Tsuchiya, H., Teshima, S., Nakanishi, H., Kwon, A.-H., Azuma, Y., Nagaoka, T., Ogawa, T., \& Kamiyama, Y. (2003). Promotive Effects of Far-Infrared Ray on Full-Thickness Skin Wound Healing in Rats. Experimental Biology and Medicine, 228(6), 724-729. https://doi.org/10.1177/153537020322800612

Tsai, SR., Hamblin, M. R. (2017). Biological effects and medical applications of infrared radiation. Journal of Photochemistry and Photobiology B: Biology, Volume 170, 197-207, https://doi.org/10.1016/j.jphotobiol.2017.04.014

Tsai, WC., Chiang, WH., Wu, CH., Li, YC., Campbell, M., Huang, PH., Lin, MW., Lin, CH., Cheng, SM., Chang, PC., Cheng, CC. (2020). miR-548aq-3p is a novel target of Far infrared radiation which predicts coronary artery disease endothelial colony forming cell responsiveness. Scientific Reports, 10, 1-17. https://doi.org/10.1038/s41598-020-63311-1

Vatansever, F. \& Hamblin, M. (2012). Far infrared radiation (FIR): Its biological effects and medical applications. Photonics $\mathcal{E}$ Lasers in Medicine, 1(4), 255-266. https:/ / doi.org/10.1515/plm-2012-0034

Yamashita, K. (2012). The Effects of the Far-Infrared Ray (FIR) Energy Radiation on Living Body, Blood Cell - An Overview of Studies in Hematology. Blood Cell - An Overview of Studies in Hematology,, https://doi.org/10.5772/36005

Yang, CC., Lin, GM., Wang, JH., Chu, HC., Wu, HT., Chen, JJ., Sun, CK. (2017). Effects of Combined Far-Infrared Radiation and Acupuncture at ST36 on Peripheral Blood Perfusion and Autonomic Activities. Evidence-Based Complementary and Alternative Medicine, Volume 2017, Article ID 1947315. https:// doi.org/10.1155/2017/1947315

Yu, SY., Chiu, JH., Yang, SD., Hsu, YC., Lui, WY., Wu, CW. (2006). Biological effect of far-infrared therapy on increasing skin microcirculation in rats. Photodermatol Photoimmunol Photomed, 22, 78-86.

$--0--$ 